\title{
Approximate Kepler's Elliptic Orbits with the Relativistic Effects
}

\author{
Leilei Jia \\ Department of Electrical Engineering, Guilin College of Aerospace Technology, Guilin, China \\ Email: jiall@guat.edu.cn
}

Received December 10, 2012; revised January 13, 2013; accepted January 21, 2013

\begin{abstract}
Beginning with a Lagrangian, we derived an approximate relativistic orbit equation which describes relativistic corrections to Keplerian orbits. The critical angular moment to guarantee the existence of periodic orbits is determined. An approximate relativistic Kepler's elliptic orbit is illustrated by numerical simulation via a second-order perturbation method of averaging.
\end{abstract}

Keywords: Kepler's Elliptic Orbits; The Relativistic Kepler Problem; Unboundedness; Averaging

\section{Introduction}

Kepler problem is one of the fundamental problems of orbital mechanics [1,2], which has been studied widely [3-5]. It is regarded as a special case of two-body problems [6], where one body is assumed to be fixed at the origin-say, for example, it is so massive, like the Sun, that to the first approximation it does not move. The Kepler's elliptic orbit is a conic section of the Kepler's equation in polar coordinates with the form

$$
r=\frac{r_{c}}{1+e \cos f},(0<e<1),
$$

where $e$ is the eccentricity and the angle $f$ is often called the true anomaly. Such elliptic orbits are of importance on describing dynamics of orbital mechanics in celestial mechanics and astrophysics.

When dealing with particles moving at speed close to that of light it may be important to take into account the relativistic effects [7-12]. There have been several attempts to obtain the orbit solution for a classical relativistic two-body system interacting electromagnetically, and the concentric circular motion of two classical relativistic point charges interacting electromagnetically had been described [13-16]. In this paper, using a perturbation techniques of averaging we will give the approximate Kepler's ellipse orbits for the Kepler problem with the special relativistic effects. In our results, we will show that once the relativistic contribution to Kepler problem is considered, the Kepler's ellipse orbit may be destroyed. However, they perhaps maintain the original characteristics for a long time.

The paper is organized as follows. Firstly, the Lagran- gian equations of motion of the relativistic Kepler problem are deduced, and the elliptic periodic orbits and unbounded orbits of equations are determined. Secondly, by the near-identity transformation, a good approximation of the Kepler's elliptic orbits is obtained via averaging of the angle. An example is given to illustrate the application of the result. Finally, we conclude our results.

\section{Periodic and Unbounded Orbits of the Relativistic Kepler Problem}

Under relativistic effects, a particle of mass $m$ orbiting a central mass $M$ is commonly described by the Lagrangian in the polar coordinates [17-21]

$$
L=-m c \sqrt{c^{2}-\dot{r}^{2}-r^{2} \dot{\theta}^{2}}-\frac{G M m}{r}
$$

where $G$ is Newton's universal gravitational constant and $c$ is the speed of light in vacuum. Then the Lagrangian equations of motion are given by

$$
\begin{aligned}
\frac{\mathrm{d}}{\mathrm{d} t} \frac{\partial L}{\partial \dot{r}} & =\frac{\partial L}{\partial r} \Rightarrow \frac{\mathrm{d}}{\mathrm{d} t} \frac{\dot{r}}{\sqrt{1-\left(\dot{r}^{2}-r^{2} \dot{\theta}^{2}\right) / c^{2}}} \\
& =\frac{r^{2} \dot{\theta}}{\sqrt{1-\left(\dot{r}^{2}-r^{2} \dot{\theta}^{2}\right) / c^{2}}}, \\
\frac{\mathrm{d}}{\mathrm{d} t} \frac{\partial L}{\partial \dot{\theta}} & =\frac{\partial L}{\partial \theta} \Rightarrow \frac{\mathrm{d}}{\mathrm{d} t} \frac{r^{2} \dot{\theta}}{\sqrt{1-\left(\dot{r}^{2}-r^{2} \dot{\theta}^{2}\right) / c^{2}}}=0 .
\end{aligned}
$$

At this moment it is convenient to introduce the relativistic linear momentum $p$ [22] 


$$
p=\frac{\dot{r}}{\sqrt{1-\left(\dot{r}^{2}-r^{2} \dot{\theta}^{2}\right) / c^{2}}}
$$

and Equation (4) implies the conservation of the relativistic angle momentum $\mu$, an arbitrary constant of integration,

$$
\mu=\frac{r^{2} \dot{\theta}}{\sqrt{1-\left(\dot{r}^{2}-r^{2} \dot{\theta}^{2}\right) / c^{2}}} .
$$

By a simply algebraic computation, we have

$$
\left\{\begin{array}{l}
\dot{r}=\frac{c r p}{\sqrt{c^{2} r^{2}+p^{2} r^{2}+\mu^{2}}}, \\
\dot{\theta}=\frac{c \mu}{r \sqrt{c^{2} r^{2}+p^{2} r^{2}+\mu^{2}}} .
\end{array}\right.
$$

Substituting (7) into (3) and together with (5), it yields that

$$
\left\{\begin{array}{l}
\dot{r}=\frac{c r p}{\sqrt{c^{2} r^{2}+p^{2} r^{2}+\mu^{2}}}, \\
\dot{p}=\frac{c \mu^{2}}{r^{2} \sqrt{c^{2} r^{2}+p^{2} r^{2}+\mu^{2}}}-\frac{G M}{r^{2}} .
\end{array}\right.
$$

Note that Equation (8) have periodic orbits if and only if the relativistic angle momentum $\mu$ is large enough, precisely, $\mu>\mu_{c}:=G M / c$. In fact, the derivation of the relativistic linear momentum $p$ always be negative when the opposite direction of inequality holds, since

$$
\begin{aligned}
\dot{p} & =\frac{1}{r^{2}}\left[\frac{c \mu}{\sqrt{1+\left(c^{2} r^{2}+p^{2} r^{2}\right) / \mu^{2}}}-G M\right] \\
& <\frac{1}{r^{2}}(\mu-G M) .
\end{aligned}
$$

For example, the mass of Sun, the Newton's universal gravitational constant and the speed of the light are taken to be $M=1.989 \times 10^{30} \mathrm{~kg}, G=6.670 \times 10^{-11} \mathrm{~m}^{3} \cdot \mathrm{kg}^{-1} \cdot \mathrm{s}^{-2}$ and $c=2.998 \times 10^{8} \mathrm{~m} \cdot \mathrm{s}^{-1}$, respectively, then the periodic orbits exist only for $\mu>4.425 \times 10^{11} \mathrm{~s}^{-1}$.

Since the change of the polar coordinates preserve the symplectic form, Equation (8) retains the Hamilton structure with the Hamiltonian

$$
H(r, p)=\frac{c}{r} \sqrt{c^{2} r^{2}+p^{2} r^{2}+\mu^{2}}-\frac{G M}{r} .
$$

The curve of level set with the Hamiltonian

$$
H(r, p)=h, h \in\left[c^{2} \sqrt{1-G^{2} M^{2} / c^{2} \mu^{2}},+\infty\right)
$$

defines the "energy" of the Kepler system (8). When

$$
h=c^{2} \sqrt{1-G^{2} M^{2} / c^{2} \mu^{2}},
$$

the curve of level set reduces to an elliptic equilibrium point

$$
\left(r^{*}, p^{*}\right)=\left(\frac{\mu}{c} \sqrt{1-\frac{G^{2} M^{2}}{c^{2} \mu^{2}}}, 0\right)
$$

of Equations (8), which is corresponding to the circle of Keplerian orbits of the form (1) with $e=0$. At the same time, every curve of level set with

$$
h \in\left[c^{2} \sqrt{1-G^{2} M^{2} / c^{2} \mu^{2}}, c^{2}\right)
$$

is corresponding to a periodic orbit of Equation (8). In case of $h \in\left[c^{2},+\infty\right)$, the orbits become unbounded and insect the $r$-axis only one time. The orbits in the phase plane $(r, p)$ for Equation (8) are depicted in Figure 1 using the parameters mentioned above.

\section{Approximate Kepler's Elliptic Orbits}

In previous section, we find that the large relativistic angle momentum $\mu$ is necessary and sufficient to guarantee the existence of the periodic orbits. At the same time, $\mu$ also as a constant of integration can be taken arbitrarily large. Consequently, in this section we will assume that $\mu$ is so large that

$$
\varepsilon=\frac{G^{2} M^{2}}{c^{2} \mu^{2}} \ll 1 .
$$

In the following, with this assumption by the method of averaging, we will show that for a long time the orbit on the $(r, \theta)$ plane is an approximate Kepler elliptic orbits. The averaged method has been used widely [23-26].

Together with (7) and (8), by successive applications of the chain rule, we get

$$
\begin{aligned}
& \frac{\mathrm{d} r}{\mathrm{~d} \theta}=\frac{\mathrm{d} r}{\mathrm{~d} t} / \frac{\mathrm{d} \theta}{\mathrm{d} t}=\frac{p r^{2}}{\mu} \Leftrightarrow \frac{\mathrm{d}}{\mathrm{d} \theta} \frac{\mu}{r}=-p, \\
& \frac{\mathrm{d} p}{\mathrm{~d} \theta}=\frac{\mathrm{d} p}{\mathrm{~d} t} / \frac{\mathrm{d} \theta}{\mathrm{d} t}=\frac{r}{\mu}-\frac{G M}{\mu} \sqrt{1+\frac{p^{2}}{c^{2}}+\frac{1}{c^{2}}\left(\frac{\mu}{r}\right)^{2}} .
\end{aligned}
$$

So it follows that

$$
\frac{\mathrm{d}^{2}}{\mathrm{~d} \theta^{2}} \frac{r_{c}}{r}+\frac{r_{c}}{r}=\sqrt{1+\varepsilon\left(\frac{\mathrm{d}}{\mathrm{d} \theta} \frac{r_{c}}{r}\right)^{2}+\left(\frac{r_{c}}{r}\right)^{2}},
$$

where $r_{c}=\mu^{2} / G M$.

Let

$$
\rho=r_{c} / r-1, \rho \in(-\infty,+\infty),
$$

then we obtain that

$$
\frac{\mathrm{d}^{2} \rho}{\mathrm{d} \theta^{2}}+\rho=\sqrt{1+\varepsilon\left[\dot{\rho}^{2}+(\rho+1)^{2}\right]}-1 .
$$




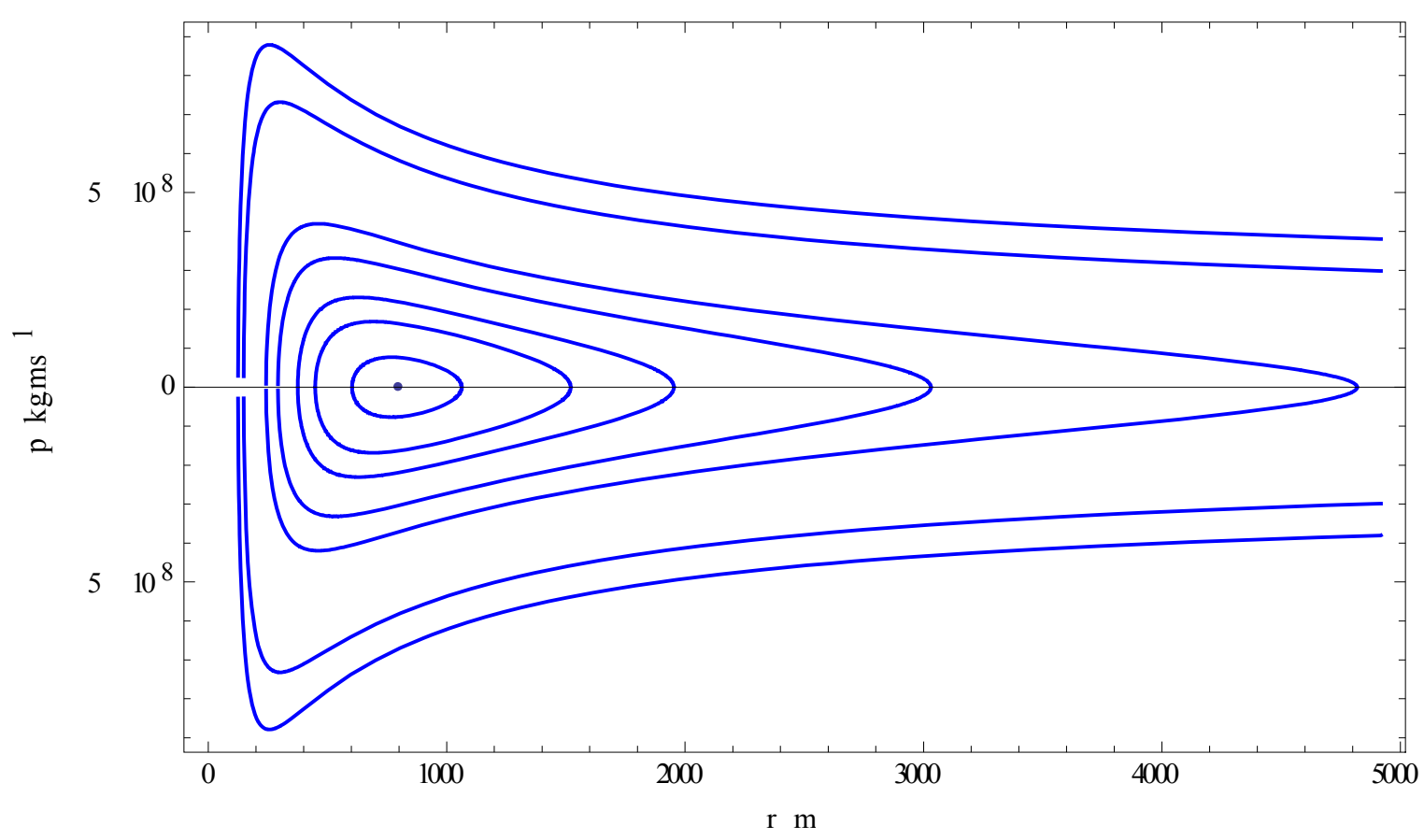

Figure 1. Periodic and unbounded orbits in the plane for the relativistic Kepler problem.

Let

$$
\rho=J \sin (\theta-\phi), \dot{\rho}=J \cos (\theta-\phi) .
$$

The perturbed Equation (13) becomes

$$
\begin{aligned}
{\left[\begin{array}{c}
\dot{J} \\
\dot{\phi}
\end{array}\right] } & =\left[\begin{array}{c}
\cos (\theta-\phi) \\
\frac{1}{J} \sin (\theta-\phi)
\end{array}\right] \times\left(\sqrt{1+\varepsilon\left[(J \cos (\theta-\phi))^{2}+(J \sin (\theta-\phi)+1)^{2}\right]}-1\right) \\
& =\varepsilon\left[\begin{array}{c}
\frac{1}{2} \cos (\theta-\phi)\left[J^{2} \cos ^{2}(\theta-\phi)+1+J^{2} \sin ^{2}(\theta-\phi)+2 J \sin (\theta-\phi)\right] \\
\frac{1}{2 J} \sin (\theta-\phi)\left[J^{2} \cos ^{2}(\theta-\phi)+1+J^{2} \sin ^{2}(\theta-\phi)+2 J \sin (\theta-\phi)\right]
\end{array}\right]+O\left(\varepsilon^{2}\right) .
\end{aligned}
$$

By the near-identity transformation

$$
\begin{gathered}
{\left[\begin{array}{l}
J \\
\phi
\end{array}\right]=\left[\begin{array}{l}
\bar{J} \\
\bar{\phi}
\end{array}\right]+\varepsilon\left[\begin{array}{l}
U^{[1]}(\bar{J}, \bar{\phi}, \theta) \\
U^{[2]}(\bar{J}, \bar{\phi}, \theta)
\end{array}\right] \quad(16) \quad \begin{array}{c}
\dot{\bar{J}}=0+O\left(\varepsilon^{2}\right), \dot{\bar{\phi}}=\frac{1}{2} \varepsilon+O\left(\varepsilon^{2}\right), \\
\text { where }
\end{array}} \\
{\left[\begin{array}{l}
U^{[1]}(\bar{J}, \bar{\phi}, \theta) \\
U^{[2]}(\bar{J}, \bar{\phi}, \theta)
\end{array}\right]=\left[\frac{1}{2} \sin (\theta-\bar{\phi})\left(1+J^{2}+J \sin (\theta-\bar{\phi})\right)-\frac{\left(1+J^{2}\right)(\cos (\theta-\bar{\phi})-1)}{2 J}-\frac{1}{4} \sin 2(\theta-\bar{\phi})\right] .}
\end{gathered}
$$

the system (15) transforms into

$$
\bar{J}(0)=e, \bar{\phi}(0)=-\pi / 2
$$

is easily solved by

$$
\dot{\bar{J}}=0+O\left(\varepsilon^{3}\right), \dot{\bar{\phi}}=\frac{1}{2} \varepsilon+\frac{1}{8} \varepsilon^{2}+O\left(\varepsilon^{3}\right) .
$$

The Equation (18) with the initial value

$$
\left\{\begin{array}{l}
\bar{J}=e+O\left(\varepsilon^{3}\right), \\
\bar{\phi}=-\frac{\pi}{2}+\frac{1}{2} \varepsilon \theta+\frac{1}{8} \varepsilon^{2} \theta+O\left(\varepsilon^{3}\right) .
\end{array}\right.
$$


Combining with the transformation (14) and (16), we have

$$
\begin{aligned}
& \frac{r_{c}}{r}=1+J \sin (\theta-\phi)=1+\left(J+\varepsilon U^{[1]}(J, \bar{\phi}, \theta)\right) \sin \left(\theta-\bar{\phi}-\varepsilon U^{[2]}(J, \bar{\phi}, \theta)\right) \\
& =1+\left\{e+\frac{\varepsilon}{2} \cos \left[\left(1-\frac{\varepsilon}{2}-\frac{\varepsilon^{2}}{8}\right) \theta\right] \times\left(1+e^{2}+e \cos \left[\left(1-\frac{\varepsilon}{2}-\frac{\varepsilon^{2}}{8}\right) \theta\right]\right)\right\} \\
& \quad \times \cos \left\{\theta-\varepsilon \frac{\theta}{2}-\varepsilon^{2} \frac{\theta}{8}-\varepsilon \frac{\left(1+\varepsilon^{2}\right)}{2 e} \sin \left[\theta-\varepsilon \frac{\theta}{2}-\varepsilon^{2} \frac{\theta}{8}\right]+\frac{\varepsilon}{4} \cos \left(2 \theta-\varepsilon \theta-\varepsilon^{2} \theta / 4\right)\right\}+O\left(\varepsilon^{2}\right) .
\end{aligned}
$$

As an example, we illustrate our results for Mercury of our solar system which is described by the near-circular orbit. Mercury has the eccentricity $(e=0.2056)$ by the classical Newton mechanics. The other parameters are taken as follows:

Newton's universal gravitational constant $G=6.670 \times$ $10^{-11} \mathrm{~m}^{3} \cdot \mathrm{kg}^{-1} \cdot \mathrm{s}^{-2}$; the mass of the Sun $M=1.989 \times 10^{30}$ $\mathrm{kg}$; the speed of light $G=2.998 \times 10^{8} \mathrm{~m} \cdot \mathrm{s}^{-1}$; the relativistic angular moment $\mu=10 \mu_{c}=10 \mathrm{GM} / \mathrm{c}$.

An approximate Kepler elliptic orbit due to special relativity is illustrated in Figure 2.

\section{Conclusion}

The relativistic angle momentum $\mu$ determines the existence of periodic orbits. When $\mu$ is smaller than the critical angle momentum $\mu_{c}$, the Kepler system (8) has no periodic orbits. For $\mu>\mu_{c}$, if the energy defined by (9) lies in a proper interval $h \in\left(c^{2} \sqrt{1-G^{2} M^{2} / c^{2} \mu^{2}}, c^{2}\right)$, then every orbit is closed and periodic; otherwise, it leads to the unbounded orbits. The approximate relativistic Kepler elliptic orbit is illustrated by numerical simulation via a second-order perturbation method of averaging, and it is valid only for timescale of the order of $1 / \varepsilon^{2}$.

\section{Acknowledgements}

This work is supported by the National Natural Science

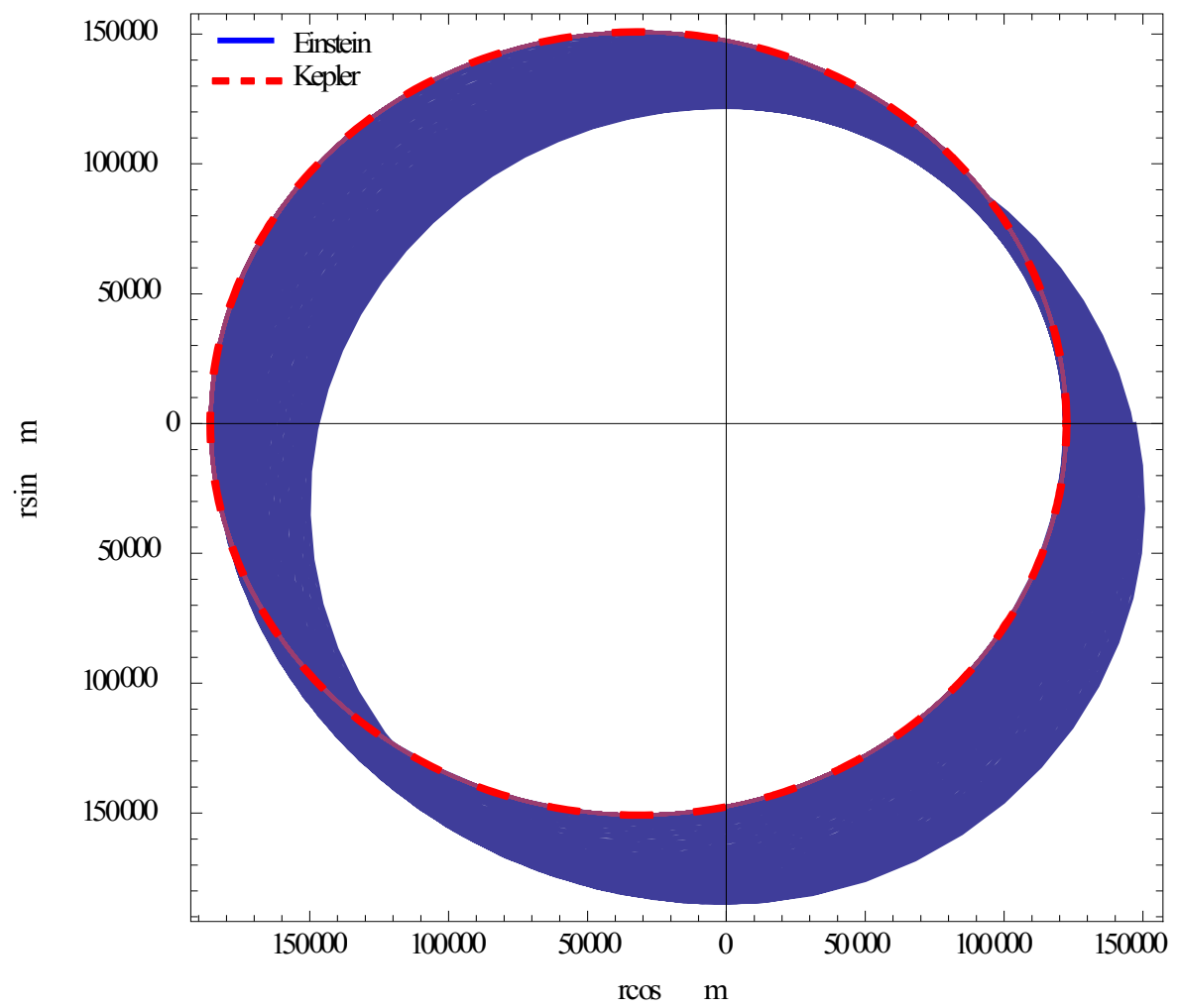

Figure 2. Relativistic orbit in a Keplerian limit (blue solid line), as described by Equation (13), compared to a corresponding Keplerian orbit (red dashed line) with $\varepsilon=\mathbf{0}$. The approximate Kepler elliptic orbit due to special relativity is illustrated here for $0 \leq \theta \leq 100 \pi$. 
Foundation of China (Grant No. 11226130 and No. 11261013).

\section{REFERENCES}

[1] R. Bate, D. Mueller and J. White, "Fundamentals of Astrodynamics," Dover Publications, New York, 1971.

[2] S. C. Bell, "A numerical Solution of the Relativistic Kepler Problem," Computers in Physics, Vol. 9, No. 3, 2001, pp. 281-285. doi:10.1063/1.168530

[3] P. Amster, J. Haddad, R. Oterga and A. J. Urena, "Periodic Motions in Forced Problems of Kepler Type," Nonlinear Differential Equations and Applications, Vol. 18, No. 6, 2011, pp. 649-657. doi:10.1007/s00030-011-0111-8

[4] A. Fonda, R. Toader, and P. J. Torres, "Periodic Motions in a Gravitational Central Field with a Rotating External Force," Celestial Mechanics and Dynamical Astronomy, Vol. 113, No. 3, 2012, pp. 335-342. doi:10.1007/s10569-012-9428-9

[5] X. GongOu, X. MingJie and Y. YaTian, "Kepler Problem in Hamiltonian Formulation Discussed from Topological Viewpoint," Chinese Physics Letters, Vol. 22, No. 7, 2005, pp. 1573-1575. doi:10.1088/0256-307X/22/7/004

[6] K. Meyer and G. Hall, "Introduction to Hamiltonian Dynamical Systems and the N-Body Problem," Springer, New York, 1992.

[7] R. G. Cawley, "Motion of a Charged Light Like Particle in an External Field," Journal of Mathematical Physics, Vol. 380, No. 14, 1967, pp. 2092-2096.

[8] T. E. Phipps, "Mercury's Precession according to Special Relativity," American Journal of physics, Vol. 54, No. 3, 1986, pp. 245-247.

[9] C. Sigismondi, "Astrometry and Relativity," Nuovo Cimento B Serie, Vol. 120, No. 10, 2005, pp. 1169-1180.

[10] N. Jun, "Unification of General Relativity with Quantum Field Theory," Chinese Physics Letters, Vol. 28, No. 11, 2011, p. 110401. doi:10.1088/0256-307X/28/11/110401

[11] F. JianHui, "Study of the Lie Symmetries of a Relativistic Variable Mass System," Chinese Physics, Vol. 11, No. 4, 2002, pp. 313-318. doi:10.1088/1009-1963/11/4/301

[12] F. J. Hui and Z. Song-Qing, "Noether's Theorem of a Rotational Relativistic Variable Mass System," Chinese Physics, Vol. 11, No. 5, 2002, pp. 445-449. doi:10.1088/1009-1963/11/5/307

[13] O. Coskun, "The Solutions of the Classical Relativistic Two-Body Equation," Turkish Journal of Physics, Vol. 22, No. 2, 2002, pp. 107-114.

[14] A. Schild, "Electromagnetic Two-Body Problem," Physi- cal Review, Vol. 131, No. 6, 1963, pp. 2762-2766. doi:10.1103/PhysRev.131.2762

[15] C. M. Andersen and H. C. Baeyer, "Circular Orbits in Classical Relativistic Two-Body Systems," Annals of Physics, Vol. 60, No. 1, 1970, pp. 67-84. doi:10.1016/0003-4916(70)90482-3

[16] P. Cordero and G. C. Ghirardi, "Dynamics for Classical Relativistic Particles: Circular Orbit Solutions and the Nonrelativistic Limit," Journal of Mathematical Physics, Vol. 14, No. 7, 1973, pp. 815-822. doi: $10.1063 / 1.1666401$

[17] T. J. Lemmon and A. R. Mondragon, "Alternative Derivation of the Relativistic Contribution to Perihelia Precession," American Journal of Physics, Vol. 77, No. 10, 2009, pp. 890-893. doi:10.1119/1.3159611

[18] J. M. Potgieter, "An Exact Solution for the Horizontal Deflection of a Falling Object," American Journal of Physics, Vol. 51, No. 3, 1983, pp. 257-258. doi:10.1119/1.13275

[19] Y. S. Huang and C. L. Lin, "A Systematic Method to Determine the Lagrangian Directly from the Equations of Motion," American Journal of Physics, Vol. 70, No. 7, 2002, pp. 741-743. doi:10.1119/1.1475331

[20] B. Coleman, "Special Relativity Dynamics without a Priori Momentum Conservation," European Journal of Physics, Vol. 26, No. 4, 2005, pp. 647-650. doi: 10.1088/0143-0807/26/4/010

[21] P. Smith and R. C. Smith, "Mechanics," John Wiley \& Sons Ltd., Chichester, 1990.

[22] P. J. Torres, A. J. Urena and M. Zamora, "Periodic and Quasi-Periodic Motions of a Relativistic Particle under a Central Force Field," Bulletin London Mathematical Society, 2012, pp. 1-13. doi: 10.1112/blms/bds076

[23] Q. Liu and D. Qian, "Construction of Modulated Amplitude Waves via Averaging in Collisionally Inhomogeneous Bose-Einstein Condensates," Journal of Nonlinear Mathematical Physics, Vol. 19, No. 2, 2012, Article ID: 1250017. doi:10.1142/S1402925112500179

[24] Q. Liu, and D. Qian, "Modulated Amplitude Waves with Nonzero Phases in Bose-Einstein Condensates," Journal of Mathematical Physics, Vol. 52, No. 8, 2011, Article ID: 082702. doi: $10.1063 / 1.3623415$

[25] L. Jia, L. Sun and J. Li, "Modulational Instability in Nonlinear Optics," Journal of Jiangxi Normal University (Natural Science Edition), Vol. 14, No. 3, 2012, pp. 271275.

[26] J. A. Sanders, F. Verhulst and J. Murdock, "Averaging Methods in Nonlinear Dynamical Systems," Springer, New York, 2007. 